\title{
Dipeptide-derived alkynes as novel irreversible inhibitors of Cathepsin B
}

Lydia Behring ${ }^{1}$, Christian Trapp ${ }^{2}$, Robert Wodtke ${ }^{1}$, Konstantin Kuhne ${ }^{1}$, Birgit Belter ${ }^{1}$, Jörg Steinbach ${ }^{1}$, Jens Pietzsch ${ }^{1}$, Reik Löser ${ }^{1}$

${ }^{1}$ Helmholtz-ZentrumDresden-Rossendorf, Germany

${ }^{2}$ Hochschule Zittau/Görlitz, Germany

https://doi.org/10.17952/35EPS.2018.064

\section{Introduction}

Until recently, alkynes were considered to be bioinert. Thus, they are popular reaction partners in bioorthogonal click reactions in vitro and in vivo. Despite the virtual chemical inertness of the alkyne moiety, two research groups observed the irreversible inhibition of a cysteine protease by an alkyne-functionalised substrate derivative: both Ekkebus et al. and Sommer et al. independently described the unexpected inactivation of deubiquitinating enzymes by ubiquitin or ubiquitin-like modifiers bearing propargylamine in place of C-terminal glycine $[1,2]$. We intended to harness these findings for the design of inhibitor-based probes for molecular functional imaging of tumourassociated cysteine proteases, particularly cathepsin B.

Tumour progression is a complex process involving an extensive number of enzymes. Amongst others, the cysteine protease cathepsin B was identified to be involved in tumour progression and metastasis. In healthy tissues, cathepsin B is located in the lysosomes, but it is secreted into the extracellular space in a broad variety of tumours. There it is, inter alia, able to degrade components of the extracellular matrix and activate different proteolytic cascades. Elevated extracellular levels are linked to increased malignancy and poor prognosis [3]. Therefore, this enzyme represents a promising target for the imaging and therapy of tumours.

The aim of our work was the synthesis and charakterisation of dipeptide-derived alkynes as novel inhibitors of cathepsin B. If the design of suitable alkyne derivatives would be successful, such inhibitors should exhibit very low unspecific reactivity, thus enabling clinical application.

Greenspan et al. reported a potent and highly selective dipeptidyl nitrile-based cathepsin B inhibitor(1, Figure 1) [4]. Based on that lead compound, a cathepsin B-targeting dipeptide alkyne was designed by isoelectronic replacement of the nitrile nitrogen atom by a methine group (2).
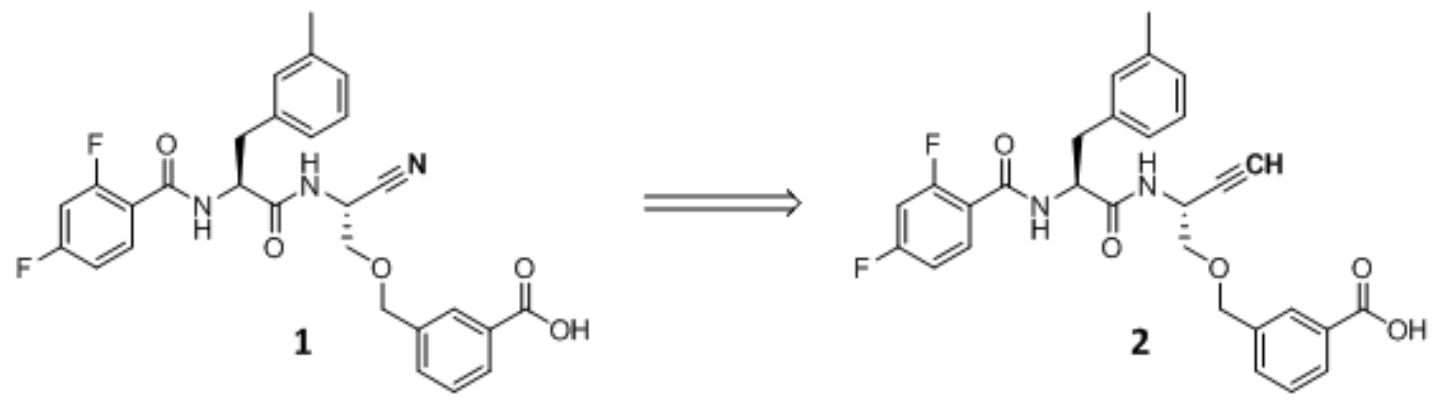

Figure 1: Inhibitor reported by Greenspan et al. (left) and irreversible inhibitor designed by isoelectronic replacement. 


\section{Results and Discussion}

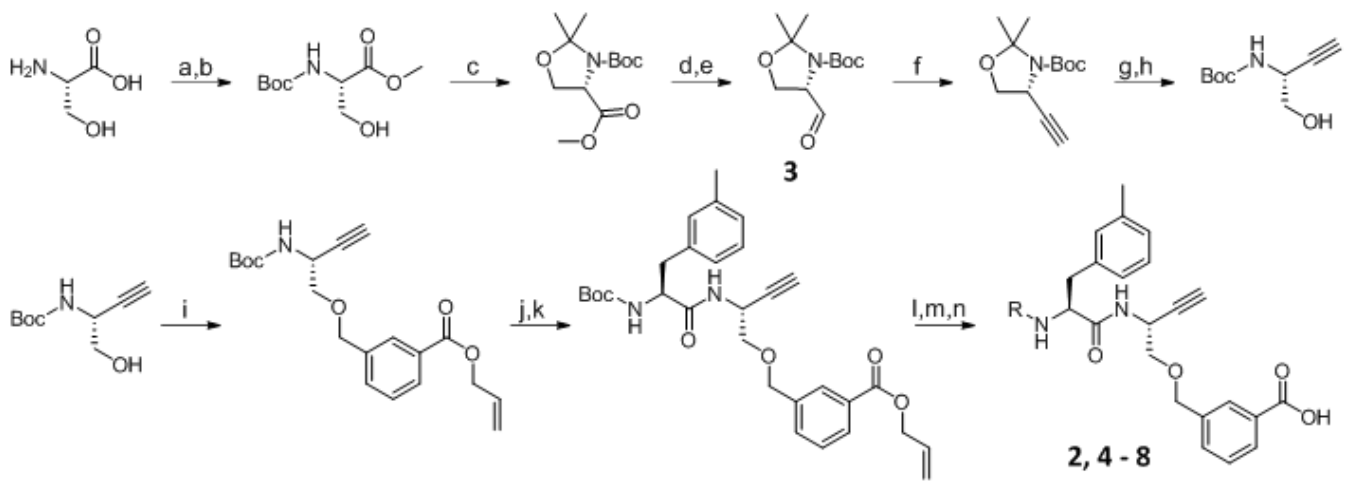

Scheme 1: Synthesis of the dipeptide-derived alkynes.

Reagents and conditions: a) acetyl chloride, $\mathrm{MeOH}$, reflux; b) Boc2O, TEA, THF; c) 2,2-dimethoxypropane, BF3. $\mathrm{OEt}_{2}$, acetone; d) $\mathrm{LiAlH}_{4}$, THF; e) oxayl chloride, DiPEA, DMSO, $\mathrm{CH}_{2} \mathrm{Cl}_{2},-78{ }^{\circ} \mathrm{C} \mathrm{rt}$; f) Bestmann-Ohira reagent, $\mathrm{K}_{2} \mathrm{CO}_{3}, \mathrm{MeOH}, 0{ }^{\circ} \mathrm{C}$ rt; g) $\mathrm{HCl}(4 \mathrm{M}), \mathrm{MeOH} / \mathrm{H}_{2} \mathrm{O}$, reflux; h) $\mathrm{Boc}_{2} \mathrm{O}$, TEA, THF; i) $\mathrm{NaH}, \mathrm{DMF}, 0{ }^{\circ} \mathrm{C}$; j) TFA, $\mathrm{CH}_{2} \mathrm{Cl}_{2}$; k) N-Boc-3-methylphenylalanine, DiPEA, PyBOP, THF; l) TFA, $\mathrm{CH}_{2} \mathrm{Cl}_{2}$; m) acyl chloride (RCl), TEA, $\mathrm{CH}_{2} \mathrm{Cl}_{2}$ or carboxylic acid (ROH), DiPEA, PyBOP, THF; n) morpholine, $\mathrm{Pd}\left(\mathrm{PPh}_{3}\right)_{4}, \mathrm{CH}_{2} \mathrm{Cl}_{2}$

In first experiments, formation of the $\mathrm{C}$ - $\mathrm{C}$ triple bond by reaction of the corresponding open-chain serine-derived aldehyde with the Bestmann-Ohira reagent was accompanied by partial enantiomerisation. Therefore, the synthesis was performed via Garner's aldehyde (3, Scheme 1). Due to conformational fixation in the fivemembered ring, enantiomerisation via deprotonation/enolisationat the $\mathrm{C}_{\alpha}$ atom was suppressed. Hence, the chosen synthesis route shown in Scheme 1 accounted for a high stereochemical purity of the final compounds.

To determine the inhibitory potential, a fluorimetric cathepsin B activity assay was p erformed. Over a time course of $15 \mathrm{~min}$ we observed the cleavage of the cathepsin B standard substrate Z-Arg-Arg-AMC $(\mathrm{Z}=$ Benzyloxycarbonyl; AMC = 7-amino-4-methylcoumarin) in the presence of different inhibitor concentrations (Figure 2A). The calculated kobs values were plotted against the inhibitor concentrations(Figure 2B) giving the specificity constant $\frac{k_{\text {inact }}}{K_{I}}$ arising from $\frac{k_{o b s}}{[I]} *\left(1+\frac{[S]}{K_{M}}\right)=\frac{k_{\text {inact }}}{K_{I}}$.
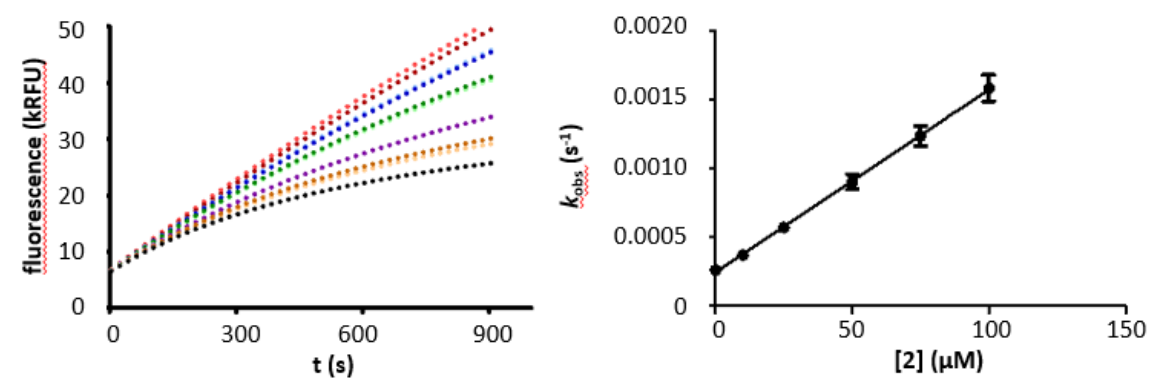

Figure 2: A) Exemplary substrate turnover curves for different concentrations of $2(0-100 \mu M)$ in the presence of cathepsin B. B) kobs versus inhibitor concentration-plot for 2 including linear regression.

Whilst the decreasing substrate turnover velocities already indicated irreversible inhibition, we performed a reactivation experiment (referred to as "jump-dilution" experiment [5]) to verify the mechanism of inhibition. As expected for reversible inhibitors, the literature-known inhibitor 1 showed complete activity recovery in the assay. In contrast, for the dipeptide-based alkyne 2 no activity recovery could be observed after dilution of the enzyme-inhibitor complex. These results proof that dipeptide-derived alkynes are indeed able to irreversibly inhibit cathepsin B.

To improve the inhibitory potential, we performed consecutive variation of the 2,4-difluorobenzoyl residue. All synthesized inhibitors were tested not only against cathepsin B, but also towards cathepsins S, L and $\mathrm{K}$ to determine their selectivity within the cathepsin family. The results are graphically depicted in Figure 
3. Compound 8 exhibited the most potent irreversible cathepsin B inhibition with an inactivation constant (kinact/KI) of $771 \mathrm{M}-1 \mathrm{~s}-1$. Values for cathepsins L and S were significantly lower; no irreversible inhibition was observed for cathepsin K. In addition, inhibition of cathepsin B activity in human glioblastoma cell lysates and living cells (U87MG and U251MG cells) has been demonstrated. Surprisingly, compound 6 turned out to be a highly potent and highly selective inhibitor for cathepsin $\mathrm{L}\left(\frac{k_{\text {inact }}}{K_{I}}=1968 \mathrm{M}^{-1} \mathrm{~S}^{-1}\right)$. As the involvement of cathepsin L in tumour progression and a correlation with poor prognosis have been demonstrated in the past [3], compound 6 will be a promising lead compound for the development of cathepsin L selective alkyne-based probes in further studies.

For values marked with " $\varnothing$ ” no irreversible inhibition could be observed within the feasible concentration range.

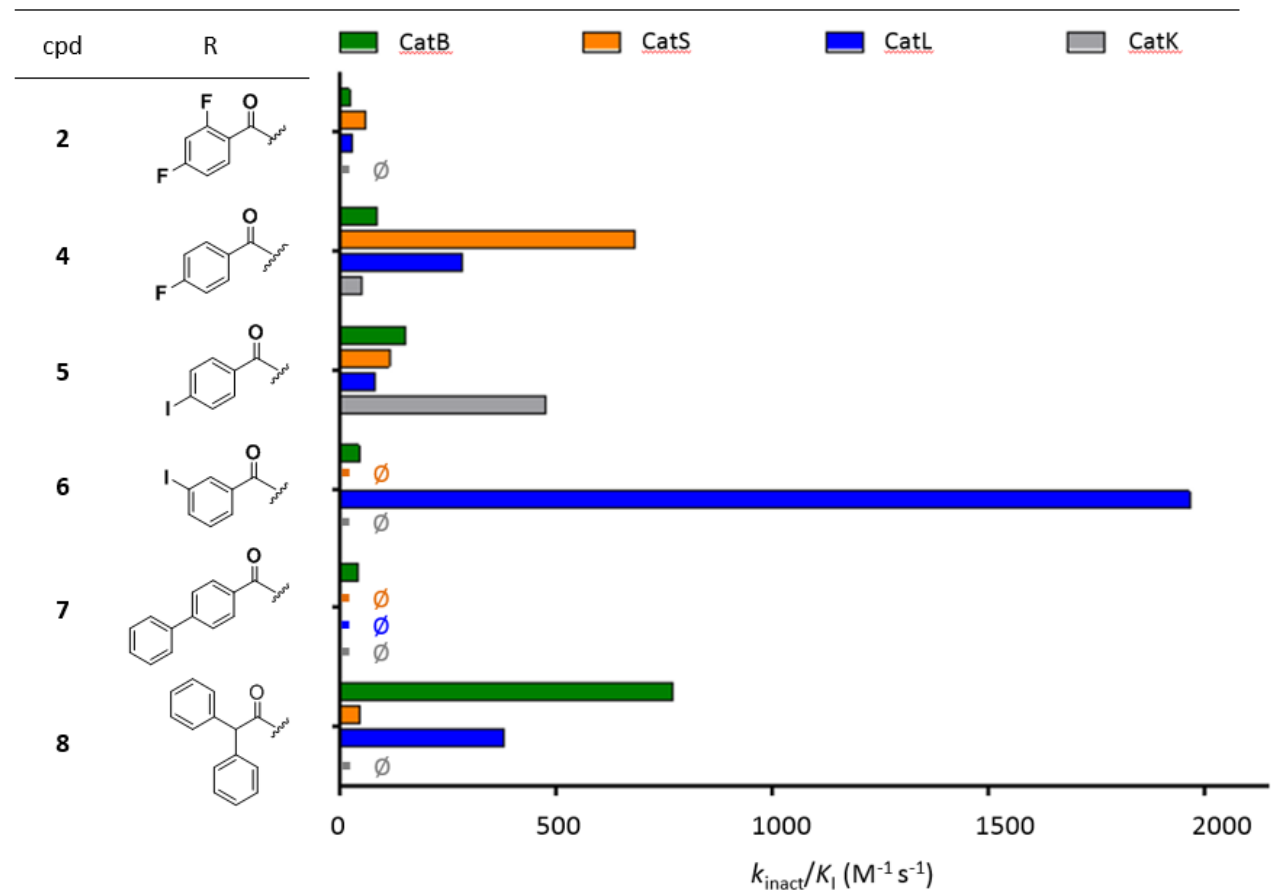

Figure 3: Selectivity profiles of assayed inhibitors with cathepsins B (CatB), S(CatS), L(CatL) and K (CatK).

\section{Conclusion}

We were able to synthesize alkyne-based irreversible inhibitors not only for cathepsin B, but also for cathepsins $\mathrm{S}, \mathrm{L}$ and $\mathrm{K}$ including a distinct selectivity profile for each enzyme by variation of the $\mathrm{N}$-terminal residue. Based on these initial results, dipeptidyl alkynes have the potential to become a valuable tool for molecular functional imaging approaches due to the expected low activity towards other cysteine proteases. In further studies, selected inhibitors for cathepsin B will be labelled with suitable radionuclides to obtain an inhibitor-based probe directed towards cathepsin B. The fact that alkynes are indeed capable to irreversibly inhibit cysteine proteases is important not only for the design of inhibitors, but also for the in vivo application of alkynes e.g. as reaction partnersin click reactions, since the possibility of unwanted side reactions must be taken into account.

\section{References}

[1] Ekkebus et al., J. Am. Chem. Soc., 2013, 135, 2867-2870.

[2] Sommer et al., Bioorg. Med. Chem., 2013, 21, 2511-2517.

[3] Löser and Pietzsch, Front. Chem., 2015, 3:37.

[4] Greenspan et al., J. Med. Chem., 2001, 44, 4524-4534.

[5] Copeland, Evaluation of Enzyme Inhibitors in Drug Discovery (2nd Edition), John Wiley \& Sons, 2013, 318-325 